\title{
Le « pendule vernaculaire » ou faire parler Arvida
}

\section{Marie-Hélène Larochelle et Samuel Archibald}

\section{(2) OpenEdition}

Journals

Édition électronique

URL : http://journals.openedition.org/edl/1218

DOI : $10.4000 /$ edl. 1218

ISSN : 2296-5084

\section{Éditeur}

Université de Lausanne

Édition imprimée

Date de publication : 15 septembre 2016

Pagination : 73-86

ISBN : 978-2-940331-49-9

ISSN : 0014-2026

Référence électronique

Marie-Hélène Larochelle et Samuel Archibald, «Le « pendule vernaculaire » ou faire parler Arvida »,

Études de lettres [En ligne], 3 | 2016, mis en ligne le 15 septembre 2019, consulté le 17 décembre 2020. URL : http://journals.openedition.org/edl/1218; DOI : https://doi.org/10.4000/edl.1218 


\section{LE «PENDULE VERNACULAIRE» OU FAIRE PARLER ARVIDA}

Notre article propose d'interroger la représentation de la langue orale québécoise dans le recueil de nouvelles Arvida (Boréal compact, 2014/Quartanier, 2011) de Samuel Archibald. Chargée d'émotion, et porteuse d'une mémoire spécifique, la voix de Samuel Archibald est aujourd'hui considérée comme l'une des plus solides dans la littérature québécoise contemporaine. La présente étude se veut originale dans sa forme puisqu'elle fera directement dialoguer le critique avec l'écrivain, qui a eu la générosité de répondre à nos questions afin d'éclairer certains aspects de son écriture. Ces fragments servent ensuite de tremplins pour produire une analyse de l'oralité dans l'écriture des récits qui composent le recueil Arvida.

Dans Le roman sans aventure, Isabelle Daunais déplore la portée limitée des romans québécois et considère que le premier problème serait $l^{\prime}$ «absence de rayonnement ${ }^{1}$ de la littérature québécoise. Elle précise: «[...] aucune de ces œuvres n'a marqué durablement l'histoire générale du roman, aucune n'est devenue, pour les lecteurs du grand contexte de l'art romanesque, une œuvre éclairante pour la compréhension de l'aventure humaine ${ }^{2}$. Trop idylliques et trop étroits, les lieux narratifs de notre littérature ne permettraient pas «de révéler et d'explorer la façon dont nous habitons le monde, dont nous l'affrontons et le concevons, dont il nous emporte» ${ }^{3}$.

La présente étude ne souhaite pas accorder trop d'importance au jugement d'Isabelle Daunais. Néanmoins, gardons en tête ses

I. I. Daunais, Le roman sans aventure, p. 7.

2. Ibid., p. 8.

3. Ibid., p. 8 . 
déclarations comme postulats avant de nous concentrer sur l'espace spécifique que constitue l'oralité, quand elle trouve sa place dans le littéraire. Il s'agira moins de s'appliquer à renverser ses hypothèses que de questionner à notre tour les possibilités de rayonnement qu'offre l'écriture québécoise.

Sous-titré "Histoires", Arvida n'est pas un roman. Il se compose de quatorze pièces, dont deux triptyques (Arvida I, II, II et Sours de sang I, II, III). Ces divers fragments se répondent et se complètent. L'unité s'établit tant grâce aux motifs privilégiés, que grâce aux personnages dont les noms et la personnalité se recoupent d'une nouvelle à l'autre. La chasse, la pêche, le bois, la bière et les automobiles les lient, animent leurs histoires, et surtout constituent des espaces de communication, des sujets de conversations qui les inspirent et les invitent à prendre la parole pour se raconter. Plusieurs Jim, Michel, se juxtaposent, sans se confondre, alors qu'une Rose-Anna fait écho à celle de Gabrielle Roy. Des Québécois, moins types humains que gens d'ici. Une cohérence se crée surtout dans les choix énonciatifs, dans la langue revendiquée, ou plutôt préférée, par les protagonistes. Français québécois, certes, mais d'abord langage comme pratique, comme travail de conversion des codes du discours, raconte Arvida.

$*$

MHL: A quel besoin de communication répondent vos textes?

SA: Il y a un personnage dans Arvida qui dit que le secret pour se souvenir de tout, c'est de ne jamais rien noter nulle part. C'est l'inverse exact de mon rapport à l'écriture. J'écris pour me débarrasser d'idées et d'histoires qui me collent aux parois crâniennes comme de la mélasse tant que je ne les écris pas.

Quant au besoin de communication, je dirais qu'il répond à un besoin, en l'endos de ma pratique de professeur et de communicateur public, de produire des messages ambigus, équivoques.

C'est ma vision profonde de la littérature que de la considérer, essentiellement, comme l'art de concevoir des propositions paradoxales, qui ne peuvent se constituer réellement que dans un dialogue, en partie agonistique, avec son destinataire. Une littérature qui aurait pour but de produire des messages clairs et limpides serait pour moi, au mieux, divertissement et, au pire, propagande. 
Cette torsion imposée au message serait donc le propre du littéraire tel que l'écrit Archibald. Prise de liberté par rapport aux exigences du schéma de la communication, l'équivoque devient le moteur de son écriture. Proche du quotidien et respectant avec une grande délicatesse les lieux du banal, Arvida n'est pourtant pas hermétique. Lambigu, si on l'observe d'abord dans les lieux de l'oral, y est subtil, prenant souvent la forme de l'hésitation, du bégaiement, propre au discours spontané. Ce naturel est une construction ciselée, une poétique qui participe au singulier Arvida.

Les trois textes sous-titrés «Arvida» I, II et III placent, par exemple, dans l'incipit la même formule d'identification «Ma grand-mère la mère de mon père" dans laquelle l'absence de ponctuation n'isole plus la précision génétique mais reconnaît plutôt le souffle de l'affection familiale. L'identité de la grand-mère est claire, tous y reconnaissent une désignation familière et d'autant plus pertinente. Cette grand-mère-ci et pas l'autre, c'est bien évident, nous sommes tous d'accord.

Car, en effet, en aval de l'écriture de l'oralité il y a le consensus. Et de ce point de vue, l'écriture d'Archibald est attachante, en ce sens qu'elle crée des liens, construit une fraternité, une amitié dans le discours. Les effets de familiarité sont essentiels puisque dans le narratif la parole ne peut être une énonciation impulsive: il faut mimer le spontané, fantasmer l'incarnation d'un sujet de l'énonciation.

La fabrication du langage narratif est un art, comme l'a établi LouisFerdinand Céline:

Encore est-ce un truc pour faire passer le langage parlé en écrit - le truc c'est moi qui l'ai trouvé personne d'autre - c'est l'impressionnisme en somme - Faire passer le langage parlé en littérature - ce n'est pas la sténographie - Il faut imprimer aux phrases, aux périodes une certaine déformation un artifice tel que lorsque vous lisez le livre il semble qu'on vous parle à l'oreille - cela s'obtient par une transposition de chaque mot qui n'est plus tout à fait celui qu'on attend une menue surprise - Il se passe ce qui aurait lieu pour un bâton plongé dans l'eau pour qu'il vous apparaisse droit il faut avant de le plonger dans l'eau que vous le cassiez légèrement si j'ose dire que vous le tordiez, préalablement ${ }^{4}$.

4. L.-F. Céline, «Lettre à M. Hindus», p. 111. 
Pour Céline, l'écriture consiste à réinventer le spontané de l'oral, à le tordre. C'est ainsi que nous lisons également l'«équivoque» d'Archibald, moins comme une obstruction que comme l'établissement d'un mouvement dans le discours écrit. Chez Louis-Ferdinand Céline, c'est la ponctuation sous forme de suspension qui s'est révélée un outil essentiel:

Mes trois points sont indispensables!... indispensables bordel Dieu!... je le répète: indispensables à mon métro! me comprenez-vous Colonel?

- Pourquoi?

- Pour poser mes rails émotifs! ${ }^{5}$

Dans Arvida, la ponctuation fait aussi l'objet d'un travail d'épuration particulier; ainsi, comme à l'oral, la conjonction est préférée à la virgule dans le récit qui ne veut pas reprendre son souffle de peur de perdre son tour de parole. Prenons le cas de ce récit très masculin d'une visite dans un club de danseuses, extrait de la nouvelle intitulée "América»:

On a bu des rhum and coke pis de la bière pis des shooters de Jameson. Lé m'a payé une danse dans l'isoloir avec la danseuse que j'avais trouvée la plus belle à date. Je sais pas combien il lui a donné, mais elle est restée avec moi au moins six chansons. J'aurais aimé ça qu'elle se frotte le cul sur moi ou qu'elle me serre la face entre ses grosses boules, mais elle arrêtait pas de me montrer sa chatte. Elle avait les petites lèvres plus grosses que les grandes pis elle arrêtait pas de jouer après pis de les étirer comme si c'était une fierté ou je sais pas quoi.

A la fin, ça m’a écouré pis j'étais gêné de regarder ${ }^{6}$.

La virilité du discours est évidente à bien des niveaux, et l'urgence du discours semble être un de ces traits essentiels. Histoire d'argent facilement gagné, histoire d'immigration illégale, mais surtout d'alliance mal organisée, la mésaventure se divise en fonction des cinq erreurs commises par la bande. L'écriture du souvenir répond dans ce récit à des conditions de réception que dévoile l'analyse de l'oralité.

Au-delà du vocabulaire proprement québécois, sur lequel nous aurons l'occasion de revenir, c'est le rythme du récit qui nous intéresse ici, car l'oralité se définit comme une dynamique, une performance ${ }^{7}$. La prise

5. L.-F. Céline, Entretiens avec le professeur $Y$, p. 114.

6. S. Archibald, Arvida, p. 97.

7. C. Vorger, Slam. 
de parole dans l'extrait du club de danseuse est de l'ordre du rapport de force: le protagoniste s'adresse dans le contexte à un public d'abord masculin dont l'intérêt est fragile, il faut maintenir leur attention au risque de ne pas avoir voix au chapitre. La parole est donc idéalement énoncée d'un seul trait pour éviter d'être interrompue. La situation de communication représentée exige le flot discursif. La virilité du discours n'est donc pas un stéréotype sexuel, mais plutôt une émotivité, un rythme dont on reconnaît les réflexes.

MHL: Votre écriture s'imposerait donc comme une forme d'archive personnelle?

SA: Comme archive personnelle et, très rapidement, communautaire. C'est bien davantage les fragments de la vie des autres, que la mienne, qui me titillent au final.

MHL: Concernant cette communauté: quelle représentation de la langue orale (québécoise, française) souhaitez-vous rendre? Quelle conscience avez-vous de cette démarche au moment de l'écriture?

SA: Il y a une partie importante de ma démarche qui consiste à me reposer constamment la question de la langue. Durant l'édition d'Arvida, j'utilisais l'expression de "pendule vernaculaire" pour désigner l'oscillation dans le recueil entre la langue blanche et très peu marquée géographiquement de certains textes, et l'oralité très très locale d'autres textes. Je pense qu'il est révélateur que mon premier livre ait été ainsi un recueil de nouvelles, qui me permettait ainsi de proposer une sorte de panorama d'usages de la langue.

Dans ma petite mystique personnelle, j’aime à me répéter que chaque histoire contient en elle-même sa propre façon d'être racontée. Je pense que c'est un trait très "québécois" que de se reposer constamment la question de la langue et d'avoir du mal à y trouver une réponse définitive. Barthes a écrit dans Critique et vérité qu'un écrivain «est quelqu'un pour qui le langage fait problème.» C'est une définition qui me semble très juste. 
La communauté qu'il met en scène, celle de la ville d'Arvida, est une communauté fantôme. Arvida est une ville industrielle fondée en 1926 qui disparaît en se fusionnant à Jonquière en 1975, qui elle-même se perdra dans la ville de Saguenay en 2002. Il y a sans aucun doute célébration d'une mémoire dans Arvida, mais plus encore, il s'agit de raviver des histoires, de les rendre actuelles et toujours pertinentes.

S'il raconte des histoires qui portent l'héritage de la Révolution tranquille, il serait réducteur de dire que Samuel Archibald écrit en joual. D'abord, parce que, comme il l'affirme lui-même, ses textes ne sont pas tous écrits dans une langue lisse et unique - certains sont très marqués par l'oralité québécoise, d'autres, peu - mais également parce que l'intégration de l'oralité qu'il privilégie est profondément moderne, actuelle, et s'écarte ainsi du débat sur la langue qui a animé les premières années de la littérature québécoise. Nous entendons par là qu'il dépasse dans une certaine mesure la "surconscience linguistique» ${ }^{8}$ identifiée par Lise Gauvin comme une préoccupation qui influence de façon particulièrement aiguë le travail des écrivains québécois.

Constamment, et surtout depuis le milieu du $\mathrm{XX}^{\mathrm{e}}$ siècle, l'auteur québécois dose la part d'oralité dans ses écrits, s'interroge sur les proportions idéales dans le contexte spécifique de son écriture, de son époque, selon un questionnement qui serait propre à la littérature québécoise. Cette sensibilité quant à la représentation de la langue orale dans la littérature touche de même le lecteur qui apprécie, et évalue, les choix de l'auteur. La littérature québécoise obligeait ainsi son récepteur à interroger le processus et les enjeux même de l'écriture de la langue française, l'invite à faire preuve d'une exigeante lucidité, voire l'inclut dans un débat perpétuellement renouvelé.

Arvida va rarement dans cette voie. Si ce n'est dans "L'animal», où survient un rare moment explicatif:

- Voulez-vous voir un our, mes bébés?

On prononçait à l'envers sur les terres du Seigneur. On disait un our et des ourses?

Il faut néanmoins préciser que, dans cette histoire, plusieurs strates sémantiques s'additionnent, et l'explication linguistique n'est qu'une part

8. L. Gauvin, Langagement, p. 8.

9. S. Archibald, Arvida p. 134. 
d'un processus de dévoilement plus global. «L'animal» raconte comment un chasseur adopte un ourson et s'attache à la bête qui ne peut plus le quitter. Epargnant la vie de l'ourson, il s'avère que le chasseur abuse de son pouvoir: il ne pourra le protéger qu'un temps, celui de l'enfance; ensuite, devenu grand, l'ours sera forcément trop sauvage pour demeurer dans le village, et il faudra l'abattre.

En parallèle, est racontée l'histoire d'une famille où un père aimant divertit ses filles, avant qu'on comprenne qu'en amont de cette affection, il y a une agression. Le vice-versa conditionne toute l'histoire, et l'explication de la prononciation inversée n'est qu'une mise en abyme d'une pratique autrement plus perturbante.

Dans Arvida, l'oralité québécoise est d'abord un accent, une intonation, une diction, et peut donc difficilement être réduite à une liste de patois propres au Saguenay ou au Québec en général. La fonction phatique domine le discours parce que le texte est un appel: le sens établit la communauté de sa réception, sans que ce lectorat ne soit exclusivement, ni même d'abord, québécois.

Ponctuellement représenté par une adresse directe, plus proche du réflexe discursif que du véritable vocare, le narrataire est un ami. Dans "Cryptozoologie», entre autres, le protagoniste raconte ses histoires de chasse et de pêche:

Leurs mordées n'ont rien de comparable avec les secousses électriques que les petites truites de ruisseau impriment sur la ligne. Au début, tu jurerais qu'un plongeur caché dans le lac a enroulé la ligne autour de son poing avant de tirer un bon coup dessus ${ }^{10}$.

Dans «América», c'est une histoire de hold-up:

Demande-moi pas comment, il a réussi à se sauver d'eux autres une demi-heure avec son vieux Topaz qui faisait le $0-100 \mathrm{~km} / \mathrm{h}$ en douze minutes à peu près. Ils ont été obligés de mettre les tapis à clous sur le boulevard des Saguenéens à la hauteur du dépanneur $247^{11}$.

Si ces deux narrateurs peuvent être identifiés comme québécois par leur syntaxe et leur vocabulaire, le narrataire qu'ils interpellent ne l'est pas forcément. Loin d'être obscure, l'oralité représentée ne pose

Io. Ibid., p. 46

II. Ibid., p. 85. 
pas de problème de réception, ne cantonne pas le texte dans des lieux québécois. Au contraire, les formules réflexes reproduites, "tu jurerais» "demande-moi pas», créent l'amitié dans le discours, forcent la connivence, et rendent le propos irrésistible selon une affection qu'on voudrait bien québécoise.

Oui, dans Arvida, ou à Arvida, on met les enfants "en pénitence» ${ }^{12}$, les femmes ont des "grosses boules" ${ }^{13}$, les enrhumés ont la "guédille au nez» ${ }^{14}$, on photographie au "kodak» ${ }^{15}$, on coince son "char» ${ }^{16}$ dans "le trafic» ${ }^{17}$, on se "chèck[e] ${ }^{18}$, on fume "une smoke» ${ }^{19}$, on sort les "vidanges» ${ }^{20}$ et on $s^{\prime}$ «enfarg[e] dans [les] planche[s] à ours» ${ }^{21}$; mais le rapport à la langue orale est chez Archibald beaucoup plus riche qu'une liste d'expressions sympathiques ou exotiques.

*

MHL: Quel rapport entretenez-vous avec le débat sur la langue au Québec? Est-ce pour vous un combat? Une façon de représenter une vérité? Une émotion (comme chez LF Céline par exemple)?

SA: J'ai été très surpris, après la publication d'Arvida, de certaines réactions, à droite et à gauche, quant à l'utilisation de la langue orale. Pour moi, c'était une affaire entendue; on peut se servir de la langue orale comme matériau de création, et c'est tout. Mais le rapport éminemment coupable, complexé et colonisé (pour employer un mot passé de mode) à la langue au Québec fait que certaines personnes voudraient sans cesse fermer le couvercle de la marmite vernaculaire sur la littérature québécoise. On semble dire «PARTI PRIS l'ont fait, Michel Tremblay l'a fait, c'était l'époque, c'était politique, maintenant, vous, les jeunes, essayez donc d'écrire comme il faut». Ça m’agace énormément, cette idée

\footnotetext{
I2. Ibid., p. 13.

I3. Ibid., p. 19.

I4. Ibid., p. 142.

I5. Ibid., p. 59.

I6. Ibid., p. 94.

17. Ibid., p. 94.

I8. Ibid., p. 89.

19. Ibid., p. 92.

2o. Ibid., p. 137.

2I. Ibid., p. 44.
} 
assez insidieusement inscrite dans la conscience nationale que l'oralité soit la manifestation d'une pauvreté (intellectuelle ou sociale).

Pour un écrivain, la langue est un instrument dont il faut savoir jouer. Il y a une volonté d'affirmation chez moi, c'est sûr. Une volonté de conservation, aussi. Et j'aime beaucoup l'idée de l'émotion (au sens célinien). Je me rappelle d'avoir pris une bière l'été dernier avec l'écrivain Patrick Nicol, que j'adore. Une partie de sa famille vient du Saguenay, du côté de sa mère, je pense. A un moment, j’ai évoqué un "saguenéisme» assez rare et difficile à écrire: un "skwâre" pour désigner un orage soudain (sans doute une déformation de l'anglais «squall»). Patrick a eu tout à coup pratiquement les larmes aux yeux et on a dû marquer une pause dans la conversation. Il n'avait pas entendu ce mot-là depuis 40 ans, dans la bouche de sa grand-mère. Il y a des vieux mots de fond de ruelle ou de fond de rang, comme ça, qui sont comme des petits mondes à eux tout seuls. J'adore m'arroger leur force d'évocation.

*

L'expression de la métaphore mérite une attention particulière. Plus que le vocabulaire, ce sont les images que construit Archibald qui font voir et entendre le Québec.

Dans "Antigonish", le narrateur voyage sur des routes sans intérêt et aboutit à l'Est sur l'océan:

Les nuages étaient gorgés d'eau comme les bâches en plastique qu'on tend au-dessus du bois à sécher, pleins d'électricité aussi. J'ai entendu un vacarme qui ressemblait plus au tonnerre qu'au ressac, j'ai vu les vagues s'entrechoquer et exploser contre les rochers dans un mouvement qui n'avait rien de doux ni d'harmonieux, j'ai vu l'océan comme une immense masse noire striée d'écume et j'ai compris que toutes les fois où j'avais vu la mer avant cette nuit-là, sur le pont d'un traversier, au phare de Pointe-au-Père ou sur la plage, à Cape Cod, j'avais vu une carte postale, j'avais vu un mensonge ${ }^{22}$.

Dans «Au milieu des araignées», il pleut également:

Derrière les grandes parois vitrées de l'aéroport, il pleuvait à boire debout. On se serait cru dans un lave-auto ${ }^{23}$. 
Dans ces deux extraits, la langue est familière, particulièrement dans le second où "il pleuvait à boire debout" (et le Québécois entend claquer le [t] final ici). Mais ce sont surtout les comparaisons qu'établissent les protagonistes qui créent une communauté de pensée. Les "bâches en plastique" gorgées d'eau, et le déluge du "lave-auto" construisent un imaginaire familier qui est moins québécois que quotidien, populaire dans une certaine mesure, et représentatif du lectorat envisagé.

Ailleurs, le lyrisme découle du choc de la langue soutenue et d'un éclat de banal. "Sur les terres du Seigneur", Rose-Anna rêve: "Ce n'était qu'une idée, une de ces idées baroques dont sont faites les insomnies et dont il ne subsiste au matin qu'une enveloppe desséchée, écorce de chêne et mue de couleuvre" ${ }^{24}$. L'imaginaire naturel et animalier parcourt toutes les histoires d'Arvida, et ses plus belles métaphores en sont teintées.

Dans «Un miroir dans le miroir», "Les pièces étaient plongées dans l'obscurité depuis si longtemps que la lumière, presque gazeuse, y pénétrait avec lenteur, en roulant sur elle-même comme une goutte de sang tombée dans l'eau" ${ }^{25}$.

Les figures d'Archibald ne sont pas spécifiquement québécoises. Le Québécois les reconnaît comme le Français ou l'Antillais. Chacun pouvant par ailleurs teinter ces images de l'accent qui lui est propre. C'est davantage un état d'esprit qu'un contexte spécifique qu'elles évoquent. Loralité, telle que nous voulons la voir dans Arvida, naît donc moins d'une accumulation de régionalismes que de l'addition de lieux communs, dont Archibald parvient à tirer les effets les plus poétiques.

Nous reconnaissons dans cette écriture la posture de l'écrivain québécois d'aujourd'hui. Jérôme Meizoz entend par "posture» l'examen des «actes énonciatifs et institutionnels complexes, par lesquels une voix et une figure se font reconnaître dans le champ littéraire " ${ }^{26}$. L'image de soi qui est donnée dans le discours respecte une mythologie populaire québécoise sans que ce contexte ne limite la réception de l'écriture. Si Samuel Archibald fait parler Arvida et entendre sa région, c'est pour que se crée une communauté de réception qui fait dialoguer le Québec avec une francophonie accueillante.

24. Ibid., p. 105.

25. Ibid., p. 123.

26. J. Meizoz, Postures littéraires, p. 11. 
MHL: Comment souhaitez-vous être lu dans le reste de la francophonie? Quel lecteur fantasmez-vous en écrivant?

SA: J'ai composé Arvida en prenant le parti de me foutre royalement de la France et de la francophonie en tant que surmoi littéraire ( $"$ mon Dieu, les Français vont-ils comprendre?»). En même temps, j’ai vécu assez longtemps là-bas pour savoir, instinctivement, jusqu'où aller trop loin dans la manifestation de la langue. Aussi, l'inscription du vernaculaire, l'ère Google, permet de transformer notre égarement en jeu de piste (j'ai beaucoup de lecteurs français qui m’ont dit s'être amusés à chercher sur Internet la signification de tel mot ou telle référence culturelle).

Je pense qu'à terme, j'ai l'objectif de dialoguer globalement avec la francophonie at large, en encourageant les usages du local au sein de la République mondiale des Lettres. Le narrateur que j'ai inventé pour mon petit roman noir Quinze pour cent manifeste bien cette volonté. J'essaye d'une certaine façon d'y mettre de l'avant une sorte de narrateur balzacien, plus modeste métaphysiquement (il se contente la plupart du temps de regarder par l'œil du personnage principal), et moins imperméable linguistiquement: à travers la conscience de Leroux, il se met souvent lui-même à inventer de nouveaux mots ou à en consigner d'anciens, et laisse parfois sa syntaxe académique être contaminée par l'oralité; tout ça, en demeurant très "lisible». Les Français trippent beaucoup sur Quinze pour cent. J'aimerais, de plus en plus, attaquer la francophonie comme lectorat sans renoncer à mon rapport archivistique et joueur à la langue parlée.

Petite note:Une chose que m’ont apprise de longs séjours en France: il n'est pas propre au Québec d'avoir un patois ou un joual. Chaque petit recoin de la francophonie a les siens. Et souvent, ils se ressemblent. C'était un grand plaisir, surtout pour les grand-parents de mes amis dans le Poitou ou le Deux-Sèvres, de m'entendre dire «barre la porte», "astheure» ou "y va mouiller tantôt». Ils disent la même chose. Ce qui est typique au Québec, c'est de croire à l'existence d'un français neutre, non marqué, radio-canadien et universellement intelligible. Ce françaislà nous «trahit» autant que l'autre.

$*$

Le combat contre les attentes de l'académisme n'a plus aujourd'hui les mêmes enjeux qu'il a pu avoir pour Michel Tremblay ou Marie-Claire 
Blais. Le "droit de mal écrire» ${ }^{27}$, pour reprendre une formule de Jérôme Meizoz, est à peu près acquis. La richesse et la polyphonie que porte l'oralité animent des écrits comme Arvida sans qu'il ne soit plus nécessaire de défendre ce choix, ou même l'usage de ce matériel. Aussi la rencontre métadiégétique que favorisaient les écrits animés d'une "surconscience linguistique» s'est-elle transformée. La voix francophone est dorénavant représentée comme un effet de présence et d'accent.

Dans le cadre de ce qu'on pourrait appeler une philosophie de la parole, le dialogisme sert de substrat pour une représentation du langage que la pragmatique nous a rendu familière: primat donné à l'énonciation, interaction discursive, rapports entre sens et contextes, historicisation radicale de la linguistique, fondement intersubjectif de l'acte de parler ${ }^{28}$.

Sortie des lieux de l'actuel, la parole devenue écriture entretient un fantasme de performance, tout en jouissant de la distance que permet le texte. Un auteur comme Archibald entretient un rapport décomplexé à l'oralité, en joue pour que résonne la dynamique alors que s'apaise le combat. Espace de voix plurielle, Arvida est ce lieu où les May West, les grilled cheese et les gâteaux usinés chantent une poétique du quotidien, libre d'un combat linguistique qui freinait aussi les possibles.

Si Isabelle Daunais regrette que "l'expérience québécoise du monde ${ }^{29}$ n'ait pas été définie par le narratif ici, qu'il nous soit maintenant permis de déplacer son hypothèse principale pour placer «l'aventure», soit «le fait pour ces derniers [les personnages] dans une situation existentielle qui les dépasse et les transforme, et, par cette expérience, de révéler un aspect jusque-là inédit ou inexploré du monde " ${ }^{30}$, au plan second pour faire primer la place de l'oralité, de l'identité linguistique, dans les représentation de l'identité québécoise dans le littéraire.

Au terme de la conversation que nous avons initiée avec Arvida, nous avons le sentiment que Samuel Archibald fait partie d'une nouvelle génération d'écrivains québécois, héritière des acquis de la Révolution tranquille, du féminisme et de la contre-culture tels qu'ils ont influencé la littérature québécoise, génération qui peut désormais jouir du dialogue

27. J. Meizoz, Le droit de "mal écrire».

28. D. Rabate, Poétique de la voix, p. 227.

29. I. Daunais, Le roman sans aventure, p. 10.

3o. Ibid., p. 15. 
qu'initie le narratif avec la parole énoncée, sans avoir à s'embarrasser du poids des processus de provocation avec lesquels devait composer la génération précédente. L'oralité représentée dans Arvida produit du sens en inscrivant les valeurs d'une communauté québécoise dont l'isolement ne s'oppose en rien au "grand contexte»; au contraire, c'est cette parole spécifique qui lui donne une épaisseur et lui permet d'y trouver sa place, sa voix.

Marie-Hélène LAROCHELLE
Université York

Samuel Archibald

Université du Québec à Montréal 


\section{BIBLIOGRAPHIE}

Archibald, Samuel, Arvida, Montréal, Boréal compact, 2014 (2011).

CÉLINe, Louis-Ferdinand, "Lettre à M. Hindus», in Michel Beaujour, Michel Thélia, Louis-Ferdinand Céline, L'Herne, 1972.

—, Entretiens avec le professeur Y, Paris, Gallimard, 1995 [1955].

Daunais, Isabelle, Le roman sans aventure, Montréal, Boréal, 2015.

Gauvin, Lise, Langagement. L'écrivain et la langue au Québec, Montréal, Boréal, 2000.

Meizoz, Jérôme, Le droit de "mal écrire». Quand les auteurs romands déjouent le "français de Paris», Genève, éd. Zoe, coll. "critique», 1998.

—, Postures littéraires. Mises en scène modernes de l'auteur. Essai, Genève, Slatkine, 2007.

Rabate, Dominique, Poétique de la voix, Paris, José Corti, coll. «les essais", 1999.

Vorger, Camille (éd.), Slam. Des origines aux horizons, Lausanne, Editions d'en bas, 2015. 